\title{
p-Adic Discrete Dynamical Systems and Collective Behaviour of Information States in Cognitive Models
}

\author{
ANDREI KHRENNIKOV* \\ Department of Mathematics, Statistics and Computer Sciences, University of Växjö, S-35195, Sweden
}

(Received 13 January 2000)

\begin{abstract}
We develop a model of functioning of complex information systems (in particular, cognitive systems) in that information states are coded by $p$-adic integers. An information state evolves by iterations of a discrete $p$-adic dynamical system. The $p$ adic utrametric on the space of information states ( $p$-adic homogeneous tree) describes the ability of an information system to operate with associations. The main attention is paid to the collective dynamics of families of associations.
\end{abstract}

Keywords: p-Adic trees; Dynamical systems; Cognitive models; Hierarchic thinning

\section{INTRODUCTION}

The system of $p$-adic numbers $\mathbf{Q}_{p}$, constructed by Hensel in the 1890s, was the first example of an infinite number field (i.e., a system of numbers where the operations of addition, subtraction, multiplication and division are well defined) which was different from a subfield of the fields of real and complex numbers. During much of the last 100 years $p$-adic numbers were considered only in pure mathematics, but in recent years they have been extensively used in theoretical physics (see, for example, the books Vladimirov et al., 1994 and Khrennikov, 1994 and the pioneer papers Volovich, 1987; Freund and Olson, 1987; Manin,
1985), the theory of probability, Khrennikov (1994) and investigations of chaos in dynamical systems Khrennikov (1997), (1998) and Albeverio et al. (1998). In Khrennikov (1998); Albeverio et al. (1999) and Dubischar et al. (1999) p-adic dynamical systems were applied to the simulation of functioning of complex information systems (in particular, cognitive systems). In this paper we continue these investigations. We study the collective dynamics of information states. We found that such a dynamics has some advantages compare to the dynamics of individual information states. First of all the use of collections of sets (instead of single points) as primary information (in particular, cognitive) units extremely extends the ability

\footnotetext{
*This research was supported by the grant "Strategical Investigations" of the University of Växjö and the visiting professor fellowship at Science University of Tokyo.
} 
of an information system to operate with large volumes of information. Another advantage is that (in the opposite to the dynamics of single states) the collective dynamics is essentially more regular. As we have seen in Khrennikov (1997); Albeverio et al. (1998), discrete dynamical systems over fields of $p$-adic numbers have the large spectrum of nonattracting behaviours. Starting with the initial point $x_{0} \in \mathbf{Q}_{p}$ iterations need not arrive to an attractor. In particular, there are numerous cycles (and cyclic behaviour depends cruicially on the prime number $p$ ) as well as Siegel disks. In our information model attractors are considered as solutions of problems (coded by initial information states $\left.x_{0} \in \mathbf{Q}_{p}\right){ }^{1}{ }^{1}$ The absence of an attractor implies that in such a model the problem $x_{0}$ could not be solved. In the opposite to dynamics of single information states ( $p$-adic numbers) collective dynamics practically always have attractors (at least for the dynamical systems which have been studied in Khrennikov, 1999 and Albeverio et al., 1998). So here each problem has the definite solution. $^{2}$

There are no physical reasons to use only prime numbers $p>1$ as the basis for the description of a physical or information model. Therefore, we use systems of so called $m$-adic numbers, where $m>1$ is an arbitrary natural number, see, for example, Mahler (1980).

\section{2. m-ADIC HIERARCHIC CHAINS FOR CODING OF INFORMATION}

The abbreviation " $I$ " will be used for information. The symbol $\tau$ will be used to denote an $I$-system.

Let $\tau$ be an $I$-system (in particular, a cognitive system). We shall use neurophysiologic terminology: elementary units for $I$-processing are called neurons, a 'thinking device' of $\tau$ is called brain. In our model it is supposed that each neuron $n$ has $m>1$ levels of excitement, $\alpha=0,1, \ldots, m-1$. Individual neurons has no $I$-meaning in this model. Information is represented by chains of neurons, $\mathcal{N}=\left(n_{0}, n_{1}, \ldots, n_{M}\right)$. Each chain of neurons $\mathcal{N}$ can (in principle) perform $m^{M}$ different $I$-states

$$
x=\left(\alpha_{0}, \alpha_{1}, \ldots, \alpha_{M-1}\right), \quad \alpha \in\{0,1, \ldots, m-1\},
$$

corresponding to different levels of excitement for neurons in $\mathcal{N}$. Denote the set of all possible $I$ states by the symbol $X_{I}$.

In our model each chain of neurons $\mathcal{N}$ has a hierarchic structure: neuron $n_{0}$ is the most important, neuron $n_{1}$ is less important than neuron $n_{0}, \ldots$, neuron $n_{j}$ is less important neurons than $n_{0}, \ldots, n_{j-1}$. This hierarchy is based on the possibility of a neuron to ignite other neurons in this chain: $n_{0}$ can ignite all neurons $n_{1}, \ldots, n_{k}, \ldots, n_{M}, \quad n_{1}$ can ignite all neurons $n_{2}, \ldots, n_{k}, \ldots, n_{M}$, and so on; but the neuron $n_{j}$ cannot ignite any of the previous neurons $n_{0}, \ldots, n_{j-1}$. Moreover, the process of igniting has the following structure. If $n_{j}$ has the highest level of excitement, $\alpha_{j}=m-1$, then increasing of $\alpha_{j}$ to one unit induces the complete relaxation of the neuron $n_{j}, \alpha_{j} \rightarrow \alpha_{j}^{\prime}=0$, and increasing to one unit of the level of excitement $\alpha_{j+1}$ of the next neuron in the chain,

$$
\alpha_{j+1} \rightarrow \alpha_{j+1}^{\prime}=\alpha_{j+1}+1
$$

If neuron $n_{j+1}$ already was maximally exited, $\alpha_{j+1}=m-1$, then transformation (2) will automatically imply the change to one unit of the state of neuron $n_{j+2}$ (and the complete relaxation of the neuron $n_{j+1}$ ) and so on. ${ }^{3}$

We shall use the abbreviation $H C N$ for hierarchic chain of neurons. This hierarchy is called a horizontal hierarchy in the I-performance in brain.

\footnotetext{
${ }^{1}$ This is more or less the standard viewpoint for models based on neural networks, see, for example, Amit (1989).

${ }^{2}$ Of course, the construction of this solution needs time and memory resources. An information system may have or may not have such resources.

${ }^{3}$ In fact, transformation (2) is the addition with respect to $\bmod m$.
} 
$H C N$ s provide the basis for forming associations. Of course, a single $H C N$ is not able to form associations. Such an ability is a feature of an ensemble $B^{\tau}$ of $H C N$ s of $\tau$. Let $s \in$ $\{0,1, \ldots, m-1\}$. A set

$$
A_{s}=\left\{x=\left(\alpha_{0}, \ldots, \alpha_{M}\right) \in X_{I}: \alpha_{0}=s\right\} \subset X_{I}
$$

is called an association of the order 1. This association is represented by a collection $B_{s}^{\tau}$ of all $H C N \mathrm{~s} \mathcal{N}=\left(n_{0}, n_{1}, \ldots, n_{M}\right)$ which have the state $\alpha_{0}=s$ for neuron $n_{0}$. Thus any association $A_{s}$ of the order 1 is represented in the brain of $\tau$ by some set $B_{s}^{\tau}$ of $H C N \mathrm{~s}$. Of course, if the set $B_{s}^{\tau}$ is empty the association $A_{s}$ does not present in the brain (at this instance of time). Associations of higher orders are defined in the same way. Let $s_{0}, \ldots, s_{l-1} \in\{0,1, \ldots, m-1\}, l \leq M$. The set

$$
\begin{aligned}
A_{s_{0} \ldots s_{l}}= & \left\{x=\left(\alpha_{0}, \ldots, \alpha_{M}\right) \in X_{I}:\right. \\
& \left.\alpha_{0}=s_{0}, \ldots, \alpha_{l-1}=s_{l-1}\right\}
\end{aligned}
$$

is called an association of the order $l$. Such an association is represented by a set $B_{s_{0} \ldots s_{l}}^{\tau} \subset B^{\tau}$ of $H C N$. We remark that associations of the order $M$ coincide with $I$-states for $H C N$. We shall demonstrate that an $I$-system $\tau$ obtains large advantages by working with associations of orders $l \ll M$.

Denote the set of all associations of order $l$ by the symbol $X_{A, l}$. We set $X_{A}=\bigcup_{l} X_{A, l}$. This is the set of all possible associations which can be formed on the basis of the $I$-space $X_{I}$.

Sets of associations $J \subset X_{A}$ also have a coginitive meaning. Such sets of associations will be called ideas of $\tau$ (of the order 1). Denote the set of all ideas by the symbol $X_{I D} \cdot{ }^{4}$ Homogeneous ideas are ideas which are formed by associations of the same order. For example, ideas $J=\left\{A_{s}, \ldots, A_{q}\right\}$, $s, \ldots, q \in\{0,1, \ldots, m-1\}$, or $J=\left\{A_{s_{1} s_{2}}, \ldots, A_{q_{1} q_{2}}\right\}$, $s_{i}, \ldots, q_{i} \in\{0,1, \ldots, m-1\}$ are homogeneous. An idea $J=\left\{A_{s}, A_{s_{1} s_{2}}, \ldots, A_{q_{1} q_{2}, \ldots, q_{l}}\right\}$ is not homogeneous. Denote the space of all ideas formed by associations of the fixed order $l$ by the symbol $X_{I D, l}$ (these ideas are homogeneous). Denote the space of all ideas formed by associations of orders less or equal to $L$ (where $L$ is the fixed number) by the symbol $X_{I D}^{L}$.

The hierarchy $I$-state $\rightarrow$ association $\rightarrow$ idea is called a vertical hierarchy in the I-performance in the brain.

Remark 2.1 (Associations, ideas and complexity of cognitive behaviour) One of the main features of our model is that not only $I$-states $x \in X_{I}$, but also associations $A \in X_{A}$ and ideas $J \in X_{I D}$ have a cognitive meaning. One of the reasons to use such a model is that complex cognitive behaviour can be demonstrated not only by living organisms $\tau$ which are able to perform in 'brains' large amounts of 'pure information' (I-states), but also by some living organisms with negligibly small 'brains'. It is well known that some primitive organisms $\tau_{\mathrm{pr}}$ having (approximately) $N=300$ nervous cells can demonstrate rather complex cognitive behaviour: ability for learning, complex sexual (even homosexual) behaviour. Suppose, for example, that the basis $m$ of the coding system of $\tau_{\mathrm{pr}}$ is equal to 2 . Here each nervous cell $n$ can yield two states: 0 , nonfiring, and 1 , firing. Nonhierarchic coding of information gives the possibility to perform in the brain (at each instance of time) 300 bits of information. In the process of 'thinking' (see Section 3) $\tau_{\mathrm{pr}}$ transforms these 300 bits into another 300 bits. It seems that such 300-bits I-dynamics could not give a complex cognitive behaviour. We now suppose that $\tau_{\mathrm{pr}}$ has the ability to create hierarchic chains of nervous cells (horisontal hierarchy). Let, for example, such $H C N$ s have the length $L=5$. Thus $\tau_{\mathrm{pr}}$ has $N=60$ $H C N$ s (so the set $B^{\tau_{\mathrm{pr}}}$ has 60 elements). The total number of $I$-states, $x=\left(\alpha_{0}, \alpha_{1}, \alpha_{2}, \alpha_{3}, \alpha_{4}\right), \alpha_{j}=0$, 1, which can be performed by $H C N$ s of the length $L=5$ is equal to $N_{I}=2^{5}=32$. Thus brain's hardware $B^{\tau_{\mathrm{pr}}}$ can perform all $I$-states (since $N_{I}<N$ ). We assume that all $I$-states are performed by the brain at each instant of time. We suppose that $\tau_{\mathrm{pr}}$

\footnotetext{
${ }^{4}$ In principle, it is possible to consider sets of ideas of the order 1 as new cognitive objects (ideas of the order 2) and so on However, we restrict our attention to dynamics of ideas of order 1 .
} 
is able to use the vertical hierarchy in the $I$ performance. The $\tau_{\mathrm{pr}}$ have $N_{a}=2^{k}$ associations of order $k=1,2, \ldots, 5$. The number of homogeneous ideas of $\tau_{\text {pr }}$ is equal

$$
\begin{aligned}
N_{I D}= & \left(2^{2}-1\right)+\left(2^{2^{2}}-1\right)+\left(2^{2^{3}}-1\right)+\left(2^{2^{4}}-1\right) \\
& +\left(2^{2^{5}}-1\right)=4295033103>>300
\end{aligned}
$$

(each term contains -1 , because empty sets of associations are not considered as ideas). Hence $\tau_{\mathrm{pr}}$ works with more than 4295033103 'ideas' (having at the same time only $N_{I}=32 I$-strings in his brain).

In our model 'hardware' of the brain of $\tau$ is given by an ensemble $B^{\tau}$ of $H C N$ s. For an $H C N$ $\mathcal{N} \in B^{\tau}$, we set $i(\mathcal{N})=x$, where $x$ is the $I$-state of $\mathcal{N}$. The map $i: B^{\tau} \rightarrow X_{I}$ gives the correspondence between states of brain and states of mind. ${ }^{5}$ In general it may be that $i\left(\mathcal{N}_{1}\right)=i\left(\mathcal{N}_{2}\right)$ for $\mathcal{N}_{1} \neq$ $\mathcal{N}_{2}$. It is natural to assume that in general the map $i$ depends on the time parameter $t: i=i_{t}$. In particular, if $t$ is discrete, we obtain a sequence of maps $i_{t}: t=0,1,2, \ldots$.

Let $O$ be some subset of $X_{I}$. The space of associations which are composed by $I$-states $x$ belonging to the set $O$ is denoted by the symbol $X_{A}(O)$. The corresponding space of ideas is denoted by the symbol $X_{I D}(O)$.

In the spatial domain model each $H C N \mathcal{N}$ is a chain of physical neurons which are connected by axons and dendrites, see, for example, Eccles (1974). In principle, such a chain of neurons can be observed (as a spatial structure in the Euclidean space $\mathbf{R}^{3}$ ), compare with Cohen et al. (1997) and Courtney et al. (1997). In the frequency domain model (see Hoppensteadt, 1997) digits $\alpha_{j} \in\{0$, $1, \ldots, m-1\}$ can be considered as (discretized) frequencies of oscillations for neurons $n_{j}, j=0,1$, $2, \ldots$, which form a 'frequency $H C N$ ' $\mathcal{N}$. Here $\mathcal{N}$ need not be imagine as a connected spatial structure. It may have a dust-like structure in $\mathbf{R}^{3}$.

\section{DYNAMICAL EVOLUTION OF INFORMATION}

In this section we shall study the simplest dynamics of $I$-states, associations and ideas. Such $I$-dynamics is "ruled" by a function $f: X_{I} \rightarrow X_{I}$ which does not depend on time and random fluctuations. This "process of thinking" has no memory: the previous $I$-state $x$ determines a new $I$-state $y$ via the transformation $y=f(x)$. In this model time is discrete, $t=0,1,2, \ldots, n, \ldots, K$. Set

$$
U_{0}^{\tau}=i_{0}\left(B^{\tau}\right), U_{1}^{\tau}=i_{1}\left(B^{\tau}\right), \ldots, U_{n}^{\tau}\left(B^{\tau}\right), \ldots
$$

A set $U_{n}^{\tau}$ of $I$-states is called an $I$-universe of $\tau$. This is the set of all $I$-states which are generated by the ensemble $B^{\tau}$ of HCNs of $\tau$ at the instant of the time $t=n$. We suppose that sets $\left\{U_{n}^{\tau}\right\}_{n=0}^{\infty}$ of $I$-states can be obtained by iterations of one fixed map $f: X_{I} \rightarrow X_{I}$. Thus dynamics (3) of $I$-universe of $\tau$ is induced by pointwise iterations:

$$
x_{n+1}=f\left(x_{n}\right) .
$$

If $x \in U_{n}^{\tau}$, then $y=f(x) \in U_{n+1}^{\tau}$. Each $x_{0} \in U_{0}^{\tau}$ evolves via $I$-trajectory: $x_{0}, \quad x_{1}=f\left(x_{0}\right), \quad x_{2}=$ $f\left(x_{1}\right)=f^{2}\left(x_{0}\right), \ldots, x_{n+1}=f\left(x_{n}\right)=f^{n}\left(x_{0}\right), \ldots$ Here the symbol $f^{n}$ denotes $n$th iteration of $f$.

Suppose that, for each association $A$, its image $B=f(A)=\{y=f(x): x \in A\}$ is again an association. Denote the class of all such maps $f$ by the symbol $\mathcal{A}\left(X_{I}\right)$. If $f \in \mathcal{A}\left(X_{I}\right)$, then dynamics (4) of $I$-states of $\tau$ induces dynamics of associations

$$
A_{n+1}=f\left(A_{n}\right) .
$$

Starting with an association $A_{0}$ (which is a subset of $I$-universe $\left.U_{0}^{\tau}\right) \tau$ obtain a sequence of associations: $A_{0}, A_{1}=f\left(A_{0}\right), \ldots, A_{n+1}=f\left(A_{n}\right), \ldots$. Dynamics of associations (5) induces dynamics of ideas: $J^{\prime}=f(J)=\left\{B^{\tau}=f(A) A \in J\right\}$. Thus each idea evolves by iterations:

$$
J_{n+1}=f\left(J_{n}\right) .
$$

\footnotetext{
${ }^{5}$ In fact, the map $i$ provides the connection between the material and mental worlds.
} 
Starting with an idea $J_{0} \tau$ obtains a sequence of ideas: $J_{0}, J_{1}=f\left(J_{0}\right), \ldots, J_{n+1}=f\left(J_{n}\right) \ldots$ In particular, by choosing $J_{0}=U_{0}^{\tau}$ we obtain dynamics of $I$-universe (which is also an idea of $\tau$ ).

We are interested in attractors of dynamical system (6) (these are ideas-solutions). To define attractors in the space of ideas $X_{I D}$, we have to define a convergence in this space. This will be done in Section 5.

\section{4. $m$-ADIC REPRESENTATION FOR INFORMATION STATES}

It is surprising that number systems which provide the adequate mathematical description of $\mathrm{HCN}$ were developed long time ago by purely number theoretical reasons. These are systems of so called $m$-adic numbers, $m>1$ is a natural numbers. First we note that in mathematical model it would be useful to consider infinite $I$-states:

$$
x=\left(\alpha_{0}, \alpha_{1}, \ldots, \alpha_{M}, \ldots\right), \quad \alpha_{j}=0,1, \ldots, m-1 .
$$

Such an $I$-state $x$ can be generated by an ideal infinite $\mathrm{HCN} \mathcal{N}$. Denote the set of all vectors (7) by the symbol $\mathbf{Z}_{m}$. This is an ideal $I$-space, $X_{I}=\mathbf{Z}_{m}$. On this space we introduce a metric $\rho_{m}$ corresponding to the hierarchic structure between neurons in chain $\mathcal{N}$ having an $I$-state $x$ : two
$I$-states $x$ and $y$ are close with respect to $\rho_{m}$ if initial (sufficiently long) segments of $x$ and $y$ coincides. If $x=\left(\alpha_{0}, \ldots, \alpha_{M}, \ldots\right), y=\left(\beta_{0}, \ldots, \beta_{M}, \ldots\right)$, and $\alpha_{0}=\beta_{0}, \ldots, \alpha_{k-1}=\beta_{k-1}$, but $\alpha_{k} \neq \beta_{k}$, then $\rho_{m}(x, y)=\left(1 / m^{k}\right)$. Such a metric is well know in number theory. This is an ultrametric: the strong triangle inequality

$$
\rho_{m}(x, y) \leq \max \left[\rho_{m}(x, z), \rho_{m}(x, y)\right]
$$

holds true. This inequality has the simple Imeaning. Let $\mathcal{N}, \mathcal{M}, \mathcal{L}$ be $\mathrm{HCNs}$ having $I$-states $x, y, z$ respectively. Denote by $k(\mathcal{N}, \mathcal{M})(k(\mathcal{N}, \mathcal{L})$ and $k(\mathcal{M}, \mathcal{L}))$ length of an initial segment in chains $\mathcal{N}$ and $\mathcal{M}(\mathcal{N}$ and $\mathcal{L}, \mathcal{M}$ and $\mathcal{L})$ such that neurons in $\mathcal{N}$ and $\mathcal{M}$ have the same level of exiting. Then is evident that

$$
k(\mathcal{N}, \mathcal{M}) \geq \min [k(\mathcal{N}, \mathcal{L}), k(\mathcal{L}, \mathcal{M})]
$$

But this gives inequality (8). As in every metric space, in $\left(\mathbf{Z}_{m}, \rho_{m}\right)$ we can introduce balls, $U_{r}(a)=\left\{x \in \mathbf{Z}_{m}: \rho_{m}(a, x) \leq r\right\}$ and spheres $S_{r}(a)=$ $\left\{x \in \mathbf{Z}_{m}: \rho_{m}(a, x)=r\right\}$ (with center at $a \in \mathbf{Z}_{m}$ of radius $r>0$ ). There is one to one correspondence between balls and associations. Let $r=\left(1 / p^{l}\right)$ and $a=\left(a_{0}, a_{1}, \ldots, a_{l-1}, \ldots\right)$. The $U_{r}(a)=\{x=$ $\left(x_{0}, x_{1}, \ldots, x_{l-1}, \ldots\right): x_{0}=a_{0}, x_{1}=a_{1}, \ldots, x_{l-1}=$ $\left.a_{l-1}\right\}=A_{a_{0} a_{1}, \ldots, a_{l-1}}$. The space of associations $X_{A}$ coincides with the space of all balls. The space of ideas $X_{I D}$ is the space which elements are families

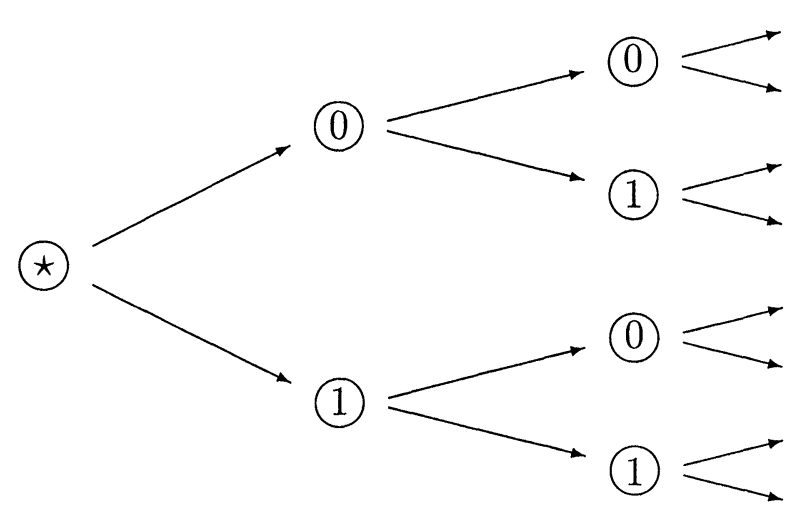

FIGURE 1 The 2-adic tree. 
of balls. Geometrically $\mathbf{Z}_{m}$ can be represented as a homogeneous tree.

Associations are represented as bundles of branches on the $m$-adic tree. Ideas are represented as sets of bundles. So dynamics (4), (5), (6) are, respectively, dynamics of branches, bundles and sets of bundles on the $m$-adic tree.

$I$-dynamics on $\mathbf{Z}_{m}$ is generated by maps $f: \mathbf{Z}_{m} \rightarrow$ $\mathbf{Z}_{m}$. We are interested in maps which belong to the class $\mathcal{A}(\mathcal{O})$, where $\mathcal{O}$ is some subset of $\mathbf{Z}_{m}$. They map a ball onto a ball: $f\left(U_{r}(a)\right)=U_{r^{\prime}}\left(a^{\prime}\right)$. To give examples of such maps, we use the standard algebraic structure on $\mathbf{Z}_{m}$.

Each sequence $x=\left(\alpha_{0}, \alpha_{1}, \ldots, \alpha_{M}, \ldots\right)$ is identified with an $m$-adic number

$x=\sum_{j=0}^{\infty} \alpha_{j} m^{j}=\alpha_{0}+\alpha_{1} \cdot m+\alpha_{2} \cdot m^{2}+\cdots+\alpha_{n} \cdot m^{n}+\cdots$.

It is possible to work with such series as with ordinary numbers. Addition, subtraction and multiplication are well defined. Analysis is much simpler for prime numbers $m=p>1$. Therefore we study mathematical models for $p$-adic numbers. Let $f_{n}(x)=x^{n}$ (n-times multiplication of $\left.x\right), n=2$, $3,4, \ldots$ We shall prove in Section 6 that $f_{n}$ belongs to the class $\mathcal{A}\left(Z_{m}^{*}\right)$, where $Z_{m}^{*}=\mathbf{Z}_{m} \mid\{0\}$. Hence here associations are transformed into associations.

$m$-adic balls $U_{r}(a)$ have an interesting property which will be used in our cognitive model. Each point $b \in U_{r}(a)$ can be chosen as a center of this ball: $U_{r}(a) \equiv U_{r}(b)$. Thus each $I$-state $x$ belonging to an association $A$ can be chosen as a base of this association. $m$-adic balls have another interesting property which will by used in our cognitive model. Let $U_{r}(a)$ and $U_{s}(b)$ be two balls. If the intersection of these balls is not empty, then one of the balls is contained in another.

\section{SEMI-METRIC SPACES OF SETS}

Let $X$ be a set. A function $\rho: X \times X \rightarrow \mathbf{R}_{+}$is said to be a metric ${ }^{6}$ if it has the following properties: (1) $\rho(x, y)=0$ iff $x=y$; (2) $\rho(x, y)=\rho(y, x)$; (3) $\rho(x, y) \leq \rho(x, z)+\rho(z, y)$ (the triangle inequality). The pair $(X, \rho)$ is called a metric space. The set $U_{r}(a)=\{x \in X: \rho(x, a) \leq r\}, a \in X, r>0$, is a ball in $X$. This set is closed in the metric space $(X, \rho)$.

A metric $\rho$ on $X$ is called an ultra-metric if the so called strong triangle inequality

$$
\rho(a, b) \leq \max [\rho(a, c), \rho(c, b)], \quad a, b, c \in X,
$$

holds true; in such a case $(X, \rho)$ is called an ultrametric space.

A distance between a point $a \in X$ and a subset $B$ of $X$ is defined as

$$
\rho(a, B)=\inf _{b \in B} \rho(a, b)
$$

(if $B$ is a finite set, then $\rho(a, B)=\min _{b \in B} \rho(a, b)$ ).

Denote by $\operatorname{Sub}(X)$ the system of all subsets of $X$. The Hausdorf distance between two sets $A$ and $B$ belonging to $\operatorname{Sub}(X)$ is defined as

$$
\rho(A, B)=\sup _{a \in A} \rho(a, B)=\sup _{a \in A} \inf _{b \in B} \rho(a, b) .
$$

If $A$ and $B$ are finite sets, then

$$
\rho(A, B)=\max _{a \in A} \rho(a, B)=\max _{a \in A} \min _{b \in B} \rho(a, b) .
$$

The Hausdorf distance $\rho$ is not a metric on the set $Y=\operatorname{Sub}(X)$. In particular, $\rho(A, B)=0$ does not imply that $A=B$. For instance, let $A$ be a subset of $B$. Then, for each $a \in A, \rho(a, B)=\inf _{b \in B} \rho(a, b)=$ $\rho(a, a)=0$. So $\rho(A, B)=0$. However, in general $\rho(A, B)=0$ does not imply $A \subset B .^{7}$ Moreover, the Hausdorf distance is not symmetric: in general $\rho(A, B) \neq \rho(B, A){ }^{8}$

\footnotetext{
${ }^{6}$ The symbol $\mathbf{R}_{+}$denotes the set of non-negative real numbers.

${ }^{7}$ Let $B$ be a non-closed subset in the metric space $X$ and let $A$ be the closure of $B$. Thus $B$ is a proper subset of $A$. Here, for each $a \in A, \rho(a, B)=\inf _{b \in B} \rho(a, b)=0$. Hence $\rho(A, B)=0$.

${ }^{8}$ Let $A \subset B$ and let $\rho(b, A) \neq 0$ at least for one point $b \in B$. Then $\rho(A, B)=0$. But $\rho(B, A) \geq \rho(b, A)>0$.
} 
We shall use the following simple fact. Let $B$ be a closed subset in the metric space $X .{ }^{9}$ Then $\rho(A, B)=0$ iff $A \subset B$. In particular, this holds true for finite sets.

The triangle inequality

$$
\rho(A, B) \leq \rho(A, C)+\rho(C, B), \quad A, B, C \in Y,
$$

holds true for the Hausdorf distance.

Let $T$ be a set. A function $\rho: T \times T \rightarrow \mathbf{R}_{+}$for that the triangle inequality holds true is called a pseudometric on $T ;(T, \rho)$ is called a pseudometric space. So the Hausdorf distance is a pseudometric on the space $Y$ of all subsets of the metric space $X$; $(Y, \rho)$ is a pseudometric space.

The strong triangle inequality

$$
\rho(A, B) \leq \max [\rho(A, C), \rho(C, B)] \quad A, B, C \in Y,
$$

holds true for the Hausdorf distance corresponding to an ultra-metric $\rho$ on $X$. In this case the Hausdorf distance $\rho$ is a ultra-pseudometric on the set $Y=\operatorname{Sub}(X)$.

We can repeat the previous considerations starting with the Hausdorf pseudometric on $Y$. We set $Z=\operatorname{Sub}(Y)$ and define the Hausdorf pseudometric on $Z$ via (11). As $\rho: Y \times Y \rightarrow \mathbf{R}_{+}$ is not a metric (and only a pseudometric) the Hausdorf pseudometric $\rho: Z \times Z \rightarrow \mathbf{R}_{+}$does not have the same properties as $\rho: Y \times Y \rightarrow \mathbf{R}_{+}$. In particular, even if $A, B \in Z=\operatorname{Sub}(Y)$ are finite sets, $\rho(A, B)=0$ does not imply that $A$ is a subset of $B$. For example, let $A=\{u\}$ and $B=\{v\}$ are singlepoint sets $(u, v \in Y=\operatorname{Sub}(X))$ and let $u \subset v$ (as subsets of $X)$. Then $\rho(u, v)=0$. If $u$ is a proper subset of $v$, then $A$ is not a subset of $B$ (in the space $Y$ ).

Proposition 5.1 Let $A, B \in Z=S u b(Y)$ be finite sets and let elements of $B$ be closed subsets of $X$. If $\rho(A, B)=0$, then, for each $u \in A$, there exists $v \in B$ such that $u \subset v$.

Proof As $\rho(A, B)=0$, then, for each $u \in A$, $\rho(u, B)=\min _{b \in B} \rho(u, b)=0$. Thus, for each $u \in A$, there exists $v \in B$ such that $\rho(u, v)=0$. As $v$ is a closed subset of $X$, this implies that $u \subset v$.

Let $A, B \in Z$ and let, for each $u \in A$, there exists $v \in B$ such that $u \subset v$. Such a relation between sets $A$ and $B$ is denoted by the symbol $A \subset \subset B$ (in particular, $A \subset B$ implies that $A \subset \subset B)$. We remark that $A \subset \subset B$ and $B \subset \subset A$ do not imply $A=B$. For instance, let $A=\left\{u_{1}, u_{2}\right\}$ and let $B=\left\{u_{2}\right\}$, where $u_{1} \subset u_{2}$. We also remark that $A_{1} \subset \subset B_{1}$ and $A_{2} \subset \subset B_{2}$ implies that $A_{1} \cup A_{2} \subset \subset B_{1} \cup B_{2}$.

Let $f: T \rightarrow T$, where $T=Y$ or $T=Z$, be a map. Let $H$ be a fixed point of $f, f(H)=H$. A basin of attraction of $H$ is the set $A T(H)=\left\{J \in T: \lim _{n \rightarrow \infty}\right.$ $\left.\rho\left(f^{n}(J), H\right)=0\right\}$. We remark that $J \in A T(H)$ means that iterations $f^{n}(J)$ of the set $J$ are (approximately) absorbed by the set $H$. The $H$ is said to be an attractor for the point $J \in Z$ if, for any fixed point $H^{\prime}$ of $f$ such that $\lim _{n \rightarrow \infty} \rho\left(f^{n}(J), H^{\prime}\right)=0$ (so $J \in A T\left(H^{\prime}\right)$ ), we have $H \subset H^{\prime}$. Thus an attractor for the set $J(\in \operatorname{Sub}(Y))$ is the minimal set that attracts $J$. The attractor is uniquely defined.

Let $T=Z=\operatorname{Sub}(Y), Y=\operatorname{Sub}(X)$. The $H$ is said to be an $\subset \subset$-attractor for the point $J \in Z$ if, for any fixed point $H^{\prime}$ of $f$ such that $\lim _{n \rightarrow \infty} \rho\left(f^{n}(J)\right.$, $\left.H^{\prime}\right)=0$ (so $J \in A T\left(H^{\prime}\right)$ ), we have $H \subset \subset H^{\prime}$. $\subset \subset$ attractor is not uniquely defined. For example, let $J=\{u\}, u \in Y, f(u)=u$. Here the set $J$ is an $\subset \subset$ attractor (for itself) as well as any refinement of $J: A=\left\{u, v_{1}, \ldots, v_{N}\right\}$, where $v_{j} \subset u$.

All previous considerations can be repeated if, instead of the spaces $Y=\operatorname{Sub}(X)$ and $Z=\operatorname{Sub}(Y)$ of all subsets, we consider some families of subsets: $U \subset \operatorname{Sub}(X)$ and $V=\operatorname{Sub}(U)$. We obtain pseudometric spaces $(U, \rho)$ and $(V, \rho)$.

Let $f: U \rightarrow U$ be a map. For $u \in U$, we set

$$
\begin{aligned}
O_{+, k}(u) & =\left\{f^{l}(u): l \geq k\right\}, k=0,1,2, \ldots, \quad \text { and } \\
O_{\infty}(u) & =\bigcap_{k=0}^{\infty} O_{+, k}(u) .
\end{aligned}
$$

For a set $J \in V$, we set $O_{+, k}(J)=\bigcup_{u \in J} O_{+, k}(u)$ and $O_{\infty}(J)=\bigcup_{u \in J} O_{\infty}(u)$.

\footnotetext{
${ }^{9} \mathrm{~A}$ closed set $B$ can be defined as a set having the property: for each $x \in X, \rho(x, B)=0$ implies that $x \in B$.
} 
Lemma 5.1 Let the space $U$ be finite. Then, for each $J \in V, J$ is attracted by the set $O_{\infty}(J)$.

Proof First we remark that, as $O_{\infty}(u) \subset \cdots \subset$ $O_{+, k+1}(u) \subset O_{+, k}(u) \cdots \subset O_{+, 0}(u)$, and $O_{+, 0}(u)$ is finite, we get that $O_{\infty}(u) \equiv O_{+, k}(u)$ for $k \geq N(u)$ (where $N(u)$ is sufficiently large).

We prove that, for each $u \in J$, the set $O_{\infty}(u)$ is $f$-invariant and

$$
\lim _{k \rightarrow \infty} \rho\left(f^{n}(u), O_{\infty}(u)\right)=0 .
$$

As $O_{\infty}(u) \equiv O_{+, k}(u), k \geq N(u)$, and $f\left(O_{+, k}(u)\right)=$ $O_{+, k+1}(u)$, we obtain that $f\left(O_{\infty}(u)\right)=O_{\infty}(u)$. If $k \geq N(u)$, then $f^{k}(u) \in O_{+, k}(u)=O_{\infty}(u)$. Thus $\rho\left(f^{k}(u), O_{\infty}(u)\right)=0$. We have

$$
f\left(O_{\infty}(J)\right)=\bigcup_{u \in J} f\left(O_{\infty}(u)\right)=\bigcup_{u \in J} O_{\infty}(u)=O_{\infty}(J) .
$$

So $O_{\infty}(J)$ is invariant. Let $N(J)=\max _{u \in J} N(u)$. If $k \geq N(J)$, then, for each $u \in J, \rho\left(f^{k}(u), O_{\infty}(J)\right) \leq$ $\rho_{p}\left(f^{k}(u), O_{\infty}(u)\right)=0$. So $J \in A T\left(O_{\infty}(J)\right)$.

A pseudometric $\rho$ (on some space) is called bounded from below if

$$
\delta=\inf \{q=\rho(a, b) \neq 0\}>0 .
$$

If $\rho$ is a metric, then (12) is equivalent to the condition

$$
\delta=\inf \{q=\rho(a, b): a \neq b\}>0 .
$$

THEOREM 5.1 Let the space $U$ be finite and let the Hausdorf distance on the space $U$ be a metric which is bounded from below. Then each set $J \in V$ has an attractor, namely the set $O_{\infty}(J)$.

Proof By Lemma 5.1 we have that $J \in A T$ $\left(O_{\infty}(J)\right)$. We need to prove that if, for some set $A \in V$,

$$
\lim _{k \rightarrow \infty} \rho\left(f^{k}(u), A\right)=0
$$

then $O_{\infty}(u) \subset A$. Let $\rho\left(f^{l}(u), A\right)<\delta$ for $l \geq k \geq N(u)$ (here $\delta$ is defined by condition (12)). As $A$ is a finite set (so $\rho(d, A)=\min _{a \in A} \rho(d, a)$ ), we obtain that

$$
\rho\left(f^{l}(u), a\right)=0
$$

for some $a=a(u, l) \in A$. Hence

$$
f^{l}(u)=a(u, l) \in A, \quad l \geq k .
$$

Thus $O_{\infty}(u)=O_{+, k}(u) \subset A$. Let

$$
\lim _{k \rightarrow \infty} \rho\left(f^{k}(J), A\right)=0
$$

As $U$ is finite (and so $J$ is also finite), (16) holds true iff (13) holds true for all $u \in J$. Thus $O_{\infty}(u) \subset A$ for each $u \in J$. So $O_{\infty}(J) \subset A$.

If the Hausdorf distance is not a metric on $U$ (and only a pseudometric), then (in general) the set $O_{\infty}(J)$ is not an attractor for the set $J$. However, we have the following result:

THEOREM 5.2 Let the space $U$ be finite and let all elements of the space $U \subset \operatorname{Sub}(X)$ be closed subsets of the metric space $(X, \rho)$. Let the Hausdorf pseudometric on the space $U$ be bounded from below. Then each set $J \in V$ has an $\subset \subset$-attractor, namely the set $O_{\infty}(J)$.

Proof By Lemma 5.1 we again have that $J \in A T\left(O_{\infty}(J)\right)$. We need to prove that if, for some set $A \in V$, (13) holds true, then $O_{\infty}(u) \subset \subset A$. We again obtain condition (14). However, as $\rho$ is just a pseudometric, this condition does not imply (15). We apply Proposition 5.1 and obtain that $f^{l}(u) \subset a(u, l)$. As $O_{\infty}(u)=O_{+, k}(u)$ for sufficently large $k$, we obtain that, for each $w \in O_{\infty}(u)$ $\left(w=f^{l}(u), l \geq k\right)$, there exists $a \in A$ such that $w \subset a$. Thus $O_{\infty}(u) \subset \subset A$.

In applications to the $I$-processing we shall use the following construction.

Let $(X, \rho)$ be an ultrametric space. We choose $U \subset \operatorname{Sub}(X)$ as the set of all balls $U_{r}(a)$. The Hausdorf distance is an ultra-pseudometric on the space of balls $U$. As balls are closed, $\rho\left(U_{r}(a)\right.$, $\left.U_{s}(b)\right)=0$ implies $U_{r}(a) \subset U_{s}(b)$. In particular, $\rho\left(U_{r}(a), U_{r}(b)\right)=0$ implies $U_{r}(a)=U_{r}(b)$. 
Proposition 5.2 Let $U_{r}(a) \cap U_{s}(b)=\emptyset . \quad$ Then $\rho\left(U_{r}(a), U_{s}(b)\right)=\rho(a, b)$.

Proof We have $\rho\left(U_{r}(a), U_{s}(b)\right) \geq \rho\left(a, U_{s}(b)\right)$. If $y \in U_{s}(b)$ then $\rho(a, b)>s \geq \rho(b, y)$. Thus $\rho(a, y)=$ $\rho(a, b)$ and, consequently, $\rho(a, b) \leq \rho\left(U_{r}(a)\right.$, $\left.U_{s}(b)\right)$. On the other hand, for each $x \in U_{r}(a)$, $\rho\left(x, U_{s}(b)\right) \leq \rho(x, b)=\rho(a, b)$. Hence $\sup _{x \in U_{r}(a)}$ $\rho\left(x, U_{s}(b)\right) \leq \rho(a, b)$.

We choose $V=\operatorname{Sub}(U)$, the space of all subsets of the space of balls.

Let $X=\mathbf{Z}_{m}$ and $\rho=\rho_{m}$. The space of associations $X_{A}$ can be identified with the space of balls $U$. Here $\rho_{m}(A, B)=0$ iff $A$ is a sub-association of $B$ : $A \subset B$. Thus $\rho_{m}\left(A_{\alpha_{0}, \ldots, \alpha_{l}}, A_{\beta_{0}, \ldots, \beta_{m}}\right)=0$ iff $l \geq s$ and $\alpha_{0}=\beta_{0}, \ldots, \alpha_{s}=\beta_{s}$. In particular, if $A, B \in X_{A, l}$ (associations of the same order $l$ ), then $\rho_{m}(A, B)=$ 0 iff $A=B$.

The space of ideas $X_{I D}$ can be identified with the space $V=\operatorname{Sub}(U)$ (of collections of balls). In such a way we introduce the Hausdorf ultrapseudometric on the space of ideas. In further constructions we shall also choose some subspaces of the space of associations $X_{A}$ and the space of ideas $X_{I D}$ as spaces $U$ and $V$, respectively.

In particular, the space $U=X_{A, l}$ of associations of the order $l$ can be identified with the space of all balls having radius $r=1 / p^{l}$. The Hausdorf distance $\rho_{m}$ is the metric on the space $U=X_{A, l}$. This metric is bounded from below with $\delta=1 / p^{l}$. So $\left(X_{A, l}, \rho_{m}\right)$ is a finite metric space with the metric (the Hausdorf distance) which is bounded from below. Theorem 5.1 can be applied for spaces $U=X_{A, l}$ and $V=X_{I D, l}=\operatorname{Sub}\left(X_{A, l}\right)$ (homogeneous ideas consisting of associations of the order $l$ ).

Theorem 5.1a Let $f: X_{I D, l} \rightarrow X_{I D, l}$ be a map induced by some map $f: X_{A, l} \rightarrow X_{A, l}$. Each idea $J \in$ $X_{I D, l}$ has an attractor, namely the set $O_{\infty}(J) \in X_{I D, l}$.

In fact, the proof of Theorem 5.1 gives the algorithm for construction of the attractor $H=O_{\infty}(J)$. The brain of a cognitive system $\tau$ produces iterations $\quad J, J_{1}=f(J), \ldots, J_{n}=$ $f\left(J_{n-1}\right), \ldots$, until the first coinsidence of a new idea $J_{s}$ with one of the previous ideas: $J_{s}=J_{n}$. As $J_{n+j}=J_{s+j}, O_{+, n}(J)=\left\{J_{n}, \ldots, J_{s-1}\right\}=O_{\infty}(J)$ is the attractor.

Remark 5.1 In fact, attractors in the space of ideas are given by cycles of associations. Dynamical systems over $p$-adic trees have a large number of cycles for $I$-states as well as for associations. This is one of the main disadvantages of the process of thinking in the domain of $I$-states and associations: starting with the initial $I$-state $x_{0}$ (or the association $A_{0}$ ) the brain of $\tau$ will often obtain no definite solution. However, by developing the ability to work with collections of associations (ideas) cognitive systems transfered this disadvantage into the great advantage: richness of cyclis behaviour on the level of associations implies richness of the set of possible ideassolutions.

Let $U=\bigcup_{l=1}^{L} X_{A, l}$. This is the collection of all associations of orders less or equal to $L$ (all balls $\left.U_{1 / p^{l}}(a), a \in \mathbf{Z}_{m}, l \leq L\right)$. Let $V=X_{I D}^{L}=\operatorname{Sub}(U)$. The Hausdorf distance is not a metric on the $U$. It is just a pseudometric: if $U_{1 / p^{l}}(a) \subset U_{1 / p^{k}}(b)$, then $\rho_{m}\left(U_{1 / p^{\prime}}(a), U_{1 / p^{k}}(b)\right)=0$. However, the Hausdorf distance is bounded from below. By Proposition 5.2 if $\rho_{m}\left(U_{1 / p^{\prime}}(a), U_{1 / p^{k}}(b)\right) \neq 0$, then $\rho_{m}\left(U_{1 / p^{\prime}}(a)\right.$, $\left.U_{1 / p^{k}}(b)\right)=\rho_{m}(a, b) \geq 1 / p^{L}$. Thus we can apply Theorem 5.2 and obtain:

Theorem 5.2a Let $f: X_{A} \rightarrow X_{A}$ be a map and let, for some $M$, the induced map $f: X_{I D}^{L} \rightarrow X_{I D}^{L}$. Then each idea $J \in X_{I D}^{M}$ has $\subset \subset$-attractor, namely the set $O_{\infty}(J) \in X_{I D}^{M}$.

As it was already been noted, $\subset \subset$-attractor is not unique. It seems that the brain of $\tau$ could have problems to determine uniquely the solution of a problem $J$. However, it would be natural for $\tau$ to produce the solution of $J$ as 'algorithmically' determined attractor $G(J) .{ }^{10}$

\footnotetext{
${ }^{10}$ We repeat again that $f: \mathbf{Z}_{m} \rightarrow \mathbf{Z}_{m}$ is not a recursive function. So we use more general viepoint to the notion of an algorithm: a recursive functions which works with nonrecursive blocks $f$. In any case we do not accept Church's thesis.
} 


\section{DYNAMICS PRESERVING THE ORDER OF ASSOCIATIONS}

We set $\mathcal{O}=S_{1}(0)$ (the unit sphere in $\mathbf{Z}_{p}$ with the center at zero). In this section we will present a large class of maps $f: \mathcal{O} \rightarrow \mathcal{O}$ which produce dynamics of associations with the property $f$ : $X_{A, m}(\mathcal{O}) \rightarrow X_{A, m}(\mathcal{O})$ for all $m$ (associations of the order $m$ are transformed into associations of the same order $m$ ).

We consider the map $f: \mathbf{Z}_{p} \rightarrow \mathbf{Z}_{p}, f=f_{n}(x)=$ $x^{n}(n=2,3, \ldots)$. The sphere $\mathcal{O}=S_{1}(0)$ is an invariant subset of this map. We shall study dynamics generated by $f$ in the $I$-space $X_{I}=\mathcal{O}$ and corresponding dynamics in spaces of associations $X_{A}(\mathcal{O})$ and $X_{I D}(\mathcal{O})$. We start with the following mathematical result:

THEOREM 6.1 The $f_{n}$-image of any ball in $Z_{p}^{*}$ is again a ball in $\boldsymbol{Z}_{p}^{*}$.

Proof Let $U_{r}(a) \subset \mathbf{Z}_{p}^{*}, r=1 / p^{m}$. As $0 \notin U_{r}(a)$, we have $|a|_{p}>r$. We shall prove that $f\left(U_{r}(a)\right)=U_{s}(b)$, where $b=a^{n}$ and $s=r|n|_{p}|a|_{p}^{n-1}$. First we prove that $f\left(U_{r}(a)\right) \subset U_{s}(b)$. Here we use the following result:

Lemma $6.1 \quad$ Let $a, \xi \in \boldsymbol{Z}_{p}$ and let $|a|_{p}>|\xi|_{p}$. Then

$$
\left|(a+\xi)^{n}-a^{n}\right|_{p} \leq|n|_{p}|a|_{p}^{n-1}|\xi|
$$

for every natural number $n$, where equality holds for $p>2$.

To prove Lemma 6.1, we use the following result, Dubischar et al. (1999):

Lemma 6.2 Let $\gamma \in \mathcal{O}$ and $u \in Z_{p},|u|_{p} \leq(1 / p)$. Then $\left|(\gamma+u)^{n}-\gamma^{n}\right|_{p} \leq|n|_{p}|u|_{p}$ for every $n \in N$, where equality holds for $p>2$.

By using (17) we obtain that $f\left(U_{r}(a)\right) \subset U_{s}(b)$. We prove that $f\left(U_{r}(a)\right)=U_{s}(b)$. Let $y=a^{n}+\beta$, where $|\beta|_{p} \leq s$. We must find $\xi,|\xi|_{p} \leq r$, such that $(a+\xi)^{n}=a^{n}+\beta$ or $(1+\xi / a)^{n}=1+\beta / a^{n}$. Formally $1+\xi / a=\left(1+\beta / a^{n}\right)^{1 / n}$. The $p$-adic binom $(1+\lambda)^{1 / n}$ is analytic for $|\lambda|_{p} \leq|n|_{p} / p$. We have $\left|\beta / a^{n}\right|_{p} \leq\left(r|n|_{p}\right) /\left(|a|_{p}\right) \leq\left(|n|_{p} / p\right)$. So $\xi=a \quad[(1+$ $\left.\left.\beta / a^{n}\right)^{1 / n}-1\right] \in \mathbf{Z}_{p}$. We have to prove that $|\xi|_{p} \leq r$.

We have

$$
\begin{aligned}
& |\xi| \leq|a|_{p} \max _{1 \leq j<\infty} \frac{|\beta|_{p}^{j}}{|n|_{p}^{j}\left|a^{n}\right|_{p}^{j}|j !|_{p}} \\
& \leq r \max _{1 \leq j<\infty}\left(\frac{r}{|a|_{p}}\right)^{j-1} \frac{1}{|j !|_{p}} .
\end{aligned}
$$

By using the inequality $\frac{1}{\mid j ! !_{p}} \leq p^{\frac{j-1}{p-1}}$, see Mahler (1980), we obtain

$$
|\xi| \leq r \max _{1 \leq j<\infty}\left(\frac{r p^{\frac{1}{p-1}}}{|a|_{p}}\right)^{j-1} \leq r .
$$

In particular, this theorem implies that

If $n$ is not divisible by $p$, the $f_{n}$-image of each ball $U_{1 / p^{m}}(a) \subset \mathcal{O}$ is a ball $U_{1 / p^{m}}(b) \subset \mathcal{O}$.

In this case $f_{n}: X_{A, m}(\mathcal{O}) \rightarrow X_{A, m}(\mathcal{O})$ for all $m$. Hence we can apply Theorems 5.1a, 5.2a. Each problem $J \in X_{I D, m}(\mathcal{O})$ has the solution $O_{\infty}(J) \in$ $X_{I D, m}(\mathcal{O})$ which is the attractor (in the space $\left.X_{I D, m}(\mathcal{O})\right)$ for $J$. Each problem $J \in X_{I D}^{M}(\mathcal{O})$ has the solution $O_{\infty}(J) \in X_{I D}^{M}(\mathcal{O})$ which is a $\subset \subset$ attractor (in the space $X_{I D}(\mathcal{O})$ ) for $J$. Moreover, the construction of the solution $O_{\infty}(J)$ can be reduced to purely arithmetical computations.

We set $R_{p^{m}}=\left\{1,2, \ldots, p^{m}-1\right\}$. We consider mod $p^{m}$ multiplication on $R_{p^{m}}$ (this is the ring of $\bmod p^{m}$ residue classes). The metric $\rho_{p}$ on $R_{p^{m}}$ is induced from $\mathbf{Z}_{p}$. This metric is bounded from below with $\delta=1 / p^{m}$. We denote by the symbol $R_{p^{m}}^{\star}$ the subset of $R_{p^{m}}$ consisting of all $j$ which are not divisible by $p$. We introduce the function $f_{n,(m)}: R_{p^{m}} \rightarrow R_{p^{m}}$ by setting $f_{n,(m)}(x)=x^{n} \bmod p^{m}$. We remark that $f_{n,(m)}$ maps the set $R_{p^{m}}^{\star}$ into itself.

Let $a \in R_{p^{m}}^{\star}$. Here set $O_{+, k}(a)=\left\{a^{n^{l}}: l \geq k\right\}$, $k=0,1,2, \ldots$, and (as usual) $O_{\infty}(a)=\bigcap_{k=1}^{\infty} O_{+, k}(a)$ and $O_{\infty}(D)=\bigcup_{d \in D} O_{\infty}(d)$ for $D \subset R_{p^{m}}^{\star}$. Let $J \in X_{I D, m}(\mathcal{O})$. So $J=\left\{U_{1 / p^{m}}(d)\right\}_{d \in D}$, where $D \subset R_{p^{m}}^{\star}$. Thus, instead of $f_{n}$-dynamics of homogeneous ideas $J \in X_{I D, m}(\mathcal{O}) \tau$ can use $f_{n,(m)^{-}}$ dynamics of collections of points $d \in R_{p^{m}}^{\star}$. It is 
performed via mod $p^{m}$ arithmetics for natural numbers. In particular, the attractor $O_{\infty}(J)=$ $\left\{U_{1 / p^{m}}(t): t \in O_{\infty}(D)\right\}$. Therefore, the solution $O_{\infty}(J)$ of the problem $J$ can constructed purely $\bmod p^{m}$-arithmetically.

CONJECTURE The process of thinking (at least its essential part) is based on $\bmod p^{m}$ arithmetics.

The same considerations can be used for nonhomogeneous ideas $J \in X_{I D}^{M}(\mathcal{O})$. Here $J=\left\{J_{m}\right\}$, where $J_{m} \in X_{I D, m}(\mathcal{O})$. Due to properties of the map $f_{n}$ all homogeneous ideas $J_{m}$ proceed independently.

\section{References}

Albeverio, S., Khrennikov, A. Yu. and Kloeden, P. (1999) Memory retrieval as a $p$-adic dynamical system. Biosystems, 49, $105-115$.

Albeverio, S., Khrennikov, A. Yu., De Smedt, S. and Tirozzi, B. (1998) p-adic dynamical systems. Theor. and Math. Phys., 114(3), 349-365.

Amit, D. J. (1989) Modeling of brain functions. Cambridge University Press, Cambridge.

Cohen, J. D., Perlstein, W. M., Braver, T. S., Nystrom, L. E., Noll, D. C., Jonides, J. and Smith, E. E., Temporal dynamics of brain activation during working memory task. Nature, 386, 604-608, April 10, 1997.
Courtney, S. M., Ungerleider, L. G., Keil, K. and Haxby, J. V., Transient and susteined activity in the disturbed neural system for human working memory. Nature, 386, 608-611, April 10, 1997.

Dubischar, D., Gundlach, V. M., Steinkamp, O. and Khrennikov, A. Yu. (1999) A $p$-adic model for the process of thinking disturbed by physiological and information noise. J. Theor. Biology, 197, 451-467.

Eccles, J. C. (1974) The understanding of the brain. McGrawHill Book Company, New-York.

Freund, P. G. O. and Olson, M. (1987) Non-Archimedean strings. Phys. Lett. B, 199, 186-190.

Hoppensteadt, F. C. (1997) An introduction to the mathematics of neurons: modeling in the frequency domain. Second edn., Cambridge University Press, New York.

Khrennikov, A. Yu. (1994) p-adic Valued Distributions in Mathematical Physics. Kluwer Academic Publishers, Dordrecht.

Khrennikov, A. Yu. (1997) Non-Archimedean analysis: quantum paradoxes, dynamical systems and biological models. Kluwer Academic Publ., Dordrecht.

Khrennikov, A. Yu. (1998) p-adic model for population growth. "Fractals in Biology and Medicine, 2." Eds. Losa, G. A., Merlini, D., Nonnemacher, T. F. and Weibel, E. R. Birkhäuser, Basel-Boston-Berlin.

Khrennikov, A. Yu. (1998) Human subconscious as a $p$-adic dynamical system. J. Theor. Biol., 193, 179-196.

Mahler, K. (1980) p-adic Numbers and their Functions. Cambridge tracts in math., 76. Cambridge Univ. Press, Cambridge.

Manin, Yu. (1985) New dimensions in geometry. Springer Lecture Notes in Math., 1111, 59-101.

Vladimirov, V. S., Volovich, I. V. and Zelenov, E. I. (1994) p-adic Analysis and Mathematical Physics. World Scientific Publ., Singapore.

Volovich, I. V. (1987) p-adic string. Class. Quant. Grav., 4, $83-87$. 


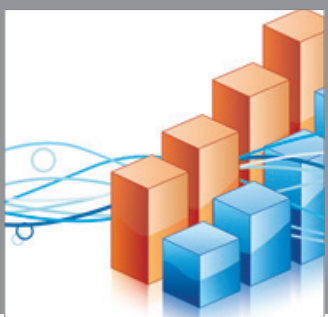

Advances in

Operations Research

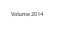

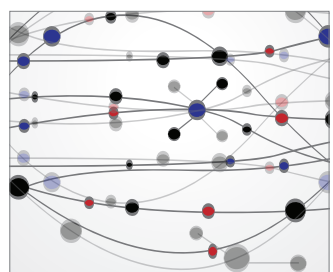

\section{The Scientific} World Journal
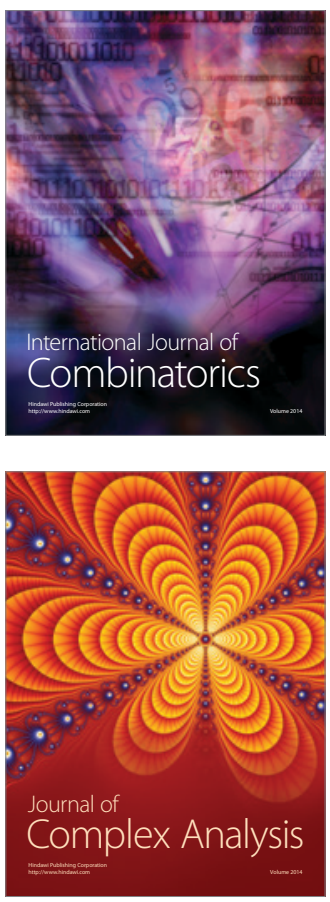

International Journal of

Mathematics and

Mathematical

Sciences
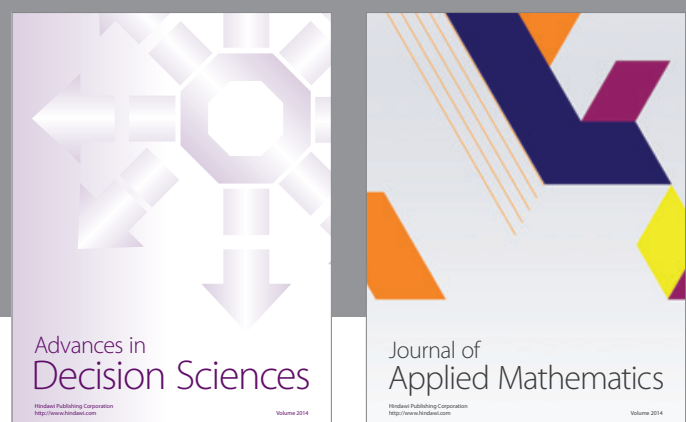

Journal of

Applied Mathematics
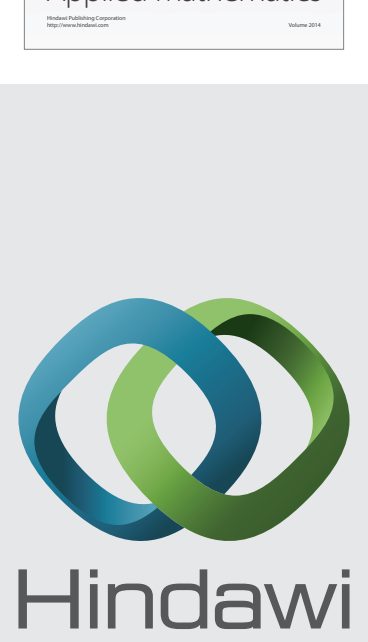

Submit your manuscripts at http://www.hindawi.com
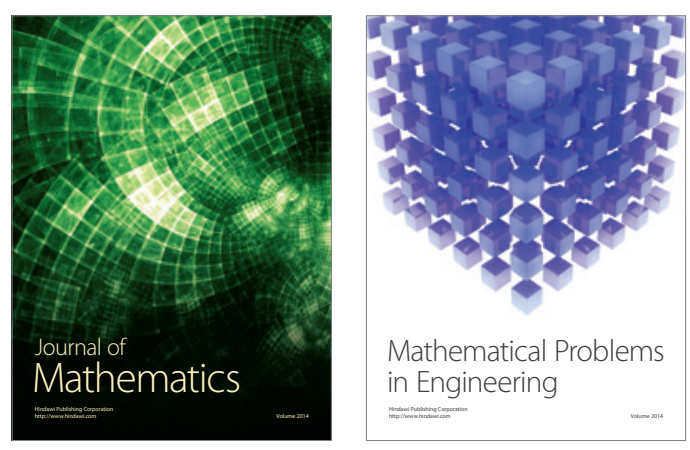

Mathematical Problems in Engineering
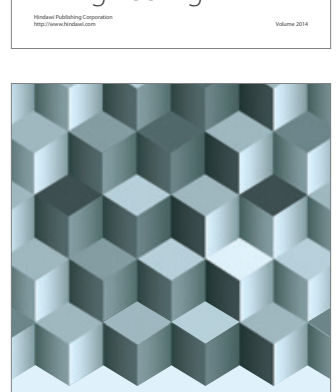

Journal of

Function Spaces
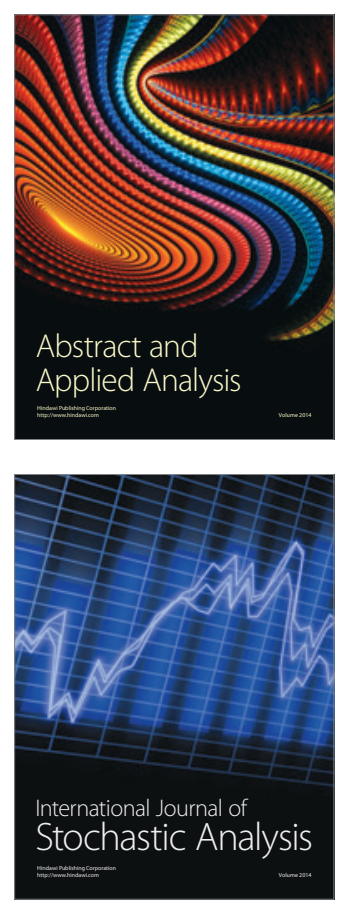

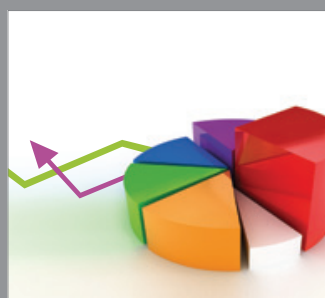

ournal of

Probability and Statistics

Promensencen
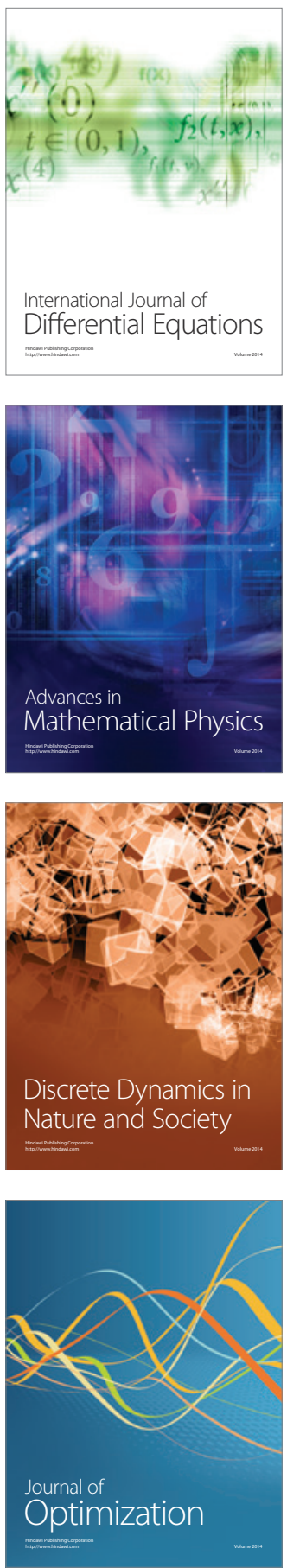